\title{
Reorganization of the Rizzoli Orthopaedic Institute during the COVID-19 outbreak
}

\author{
C. Faldini ${ }^{1}$
}

Published online: 18 November 2020

(C) Istituto Ortopedico Rizzoli 2020

Since its identification in Wuhan (China) and the report to the international community in December 2019, the severe acute respiratory syndrome coronavirus 2 (SARS-Cov-2) has spread globally, resulting in the COVID-19 pandemic [1,2].

In February 2020, the COVID-19 outbreak started in Northern Italy, gradually involving the whole country, and so far the number of affected patients has reached more than 790,000 COVID cases [3-5].

Since the beginning of the outbreak an overload of city hospitals has been observed in the whole country, challenging the National Health System (NHS). The Italian NHS is public and delivers universal coverage for medical and hospital care [6]. During the COVID-19 pandemic, the NHS has to deal with several issues: the urgent need for a higher number of Intensive Care Unit (ICU) beds, and more than usual standard hospital beds to manage ill patients with respiratory symptoms [7]; moreover, a need to rearrange the organization and resource distribution inside hospitals and in the metropolitan areas has been the main task of the crisis units of the NHS on a local basis [5].

The viral spread followed different patterns in distinct geographic areas in Italy. Bologna is the capital city of Emilia Romagna and hosts the Rizzoli Institute (RI), the oldest orthopedic institute in the world, that was founded in 1896 by Francesco Rizzoli; it is a musculoskeletal surgery facility dedicated to the management of complex orthopedic surgical cases (tumors, infections, complex spine and revision surgery), most of which are performed as elective surgery, while trauma casualties account for less than $30 \%$ of its regular surgical activity [6]. Since the beginning of the COVID-19 pandemic, the RI has been converted into a trauma center for the management of patients in the metropolitan area. In the past decades, a similar reorganization

$\triangle$ C. Faldini

cesare.faldini@ior.it

1 1st Orthopaedic Clinic, IRCCS - Istituto Ortopedico Rizzoli, DIBINEM - University of Bologna, Bologna, Italy occurred another two times, during world wars 1 and 2, when the hospital was directed by Vittorio Putti and Francesco Delitala, respectively, responding to the needs of the community to manage war wounds, fractures and amputees.

During the COVID emergency, the clinical protocols and surgical activity of the RI have been modified to support the current needs of the NHS. The Emergency Department has now two different pathways and areas for the management of standard and suspected/positive COVID cases. Moreover, a separate ward dedicated to COVID patients holds positive trauma patients admitted from the Emergency Department; it also serves to continue care of patients becoming symptomatic or diagnosed after hospital admission. A separated operating theater, once devoted to the management of infections of the musculoskeletal system, is now used for the management of COVID patients only. At present, most of the trauma cases of the metropolitan area are referred to the RI, except for polytraumas. In this way, the other hospitals of the city of Bologna have more beds and personnel that can be reassigned to the management of COVID casualties, if needed.

The rearrangement of the RI has been fast, even though not all changes were made at the same time, and current protocols are the result of a continuous process of reorganization. Seen the novelty of the disease, it is not possible to determine an approximate date of emergency ending. After resolution of the national lockdown, a progressive reopening of all the working activities had occurred, but beginning at the end of summer, the second wave of the disease has started: in this scenario, a progressive reorganization to restart operating on elective patients in a controlled infective risk scenario is quintessential.

Differently from the first wave of the disease, actual protocols allow the performance of elective orthopedic surgery, crucial to provide the continuity of care to patients, since it is not possible to estimate when the pandemic will end. Restrictive measures including regionally differentiated lockdowns and strict application of general hygiene rules can 
be effective in breaking down the infection, and in flattening the curve of the pandemic [3]; however, an unpredictable number of COVID patients is expected over a long period of time, potentially with several waves of contagion $[8,9]$.

At the time of COVID-19 pandemic, finding a compromise is the key to provide elevated standards of care with an acceptable infective risk: the hospitalization of elective patients can in fact be rationalized and regulated to keep the infection risk as low as possible both for patients and healthcare professionals: a progressive resumption of the volume of surgery, with a gradual increase of number of patients, respecting the health policies of social distancing, may keep the infective risk under control. Moreover, in relation to the uncertain effects of the restrictive measures on virus spread, it is recommended to keep an adequate availability of health resources for COVID casualties. (i.e., at least $30 \%$ of intensive care beds free and immediately available).

The current period is the benchmark to evaluate the safety and effectiveness of the current strategy and, at the same time, it will be used to evaluate health areas that best manage the elective activity while controlling COVID casualties [10].

Staff care is important as much as patient safety, and current figures show that health professionals are at risk during COVID-19 patient care [3]. Continuous training of health practitioners about hygiene rules and appropriate use of PPE, as well as advanced personnel safety protocols and effective epidemiological management of COVID casualties among health practitioners are required: surveillance of contacts between staff members, strictly managed by the occupational medicine unit, is of paramount importance to minimize and keep under control infection rate in the hospital.

Rearrangement of hospital organization and workflows, as performed at the RI, is the base for the development of protocols to continue performing elective orthopedic surgery while keeping the infectious risk as low as possible for both the patients and caregivers. Protocols for resumption of elective surgical activities must be evidence-based, but flexible enough to change on the base of the local framework.

\section{Compliance with ethical standards}

Conflict of interest There is no conflict of interest.

\section{References}

1. Cucinotta D, Vanelli M (2020) WHO declares COVID-19 a pandemic. Acta Biomed 91:157-160. https://doi.org/10.23750/abm. v91i1.9397

2. Coronavirus disease (COVID-19) - World Health Organization. https://www.who.int/emergencies/diseases/novel-coronaviru s-2019. Accessed 5 Nov 2020

3. EpiCentro Coronavirus I Istituto Superiore di Sanità. https://www. epicentro.iss.it/en/coronavirus/. Accessed 5 Nov 2020

4. Livingston E, Bucher K (2020) Coronavirus disease 2019 (COVID-19) in Italy. JAMA. https://doi.org/10.1001/ jama.2020.4344

5. Paterlini M (2020) On the front lines of coronavirus: the Italian response to covid-19. BMJ 368:m1065. https://doi.org/10.1136/ bmj.m1065

6. Di Martino A, Faldini C (2020) Trauma Service reorganization in Bologna (Italy) during COVID-19 pandemic. Injury. https://doi. org/10.1016/j.injury.2020.04.033

7. Grasselli G, Pesenti A, Cecconi M (2020) Critical care utilization for the COVID-19 outbreak in Lombardy, Italy: early experience and forecast during an emergency response. JAMA. https://doi. org/10.1001/jama.2020.4031

8. Hatchett RJ, Mecher CE, Lipsitch M (2007) Public health interventions and epidemic intensity during the 1918 influenza pandemic. Proc Natl Acad Sci USA 104:7582-7587. https://doi. org/10.1073/pnas.0610941104

9. Vannabouathong C, Devji T, Ekhtiari S et al (2020) Novel coronavirus COVID-19: current evidence and evolving strategies. J Bone Joint Surg Am. https://doi.org/10.2106/JBJS.20.00396

10. Sun P, Lu X, Xu C et al (2020) Understanding of COVID-19 based on current evidence. J Med Virol. https://doi.org/10.1002/ jmv. 25722

Publisher's Note Springer Nature remains neutral with regard to jurisdictional claims in published maps and institutional affiliations. 